\title{
El playground y las propuestas para la educación de los niños (desde la Infant School hasta el Parque Infantil, 1823-1935)*
}

The Playground and Proposals for Children's Education (From Infant School to Parque Infantil, 1823-1935)

O playground e as propostas para a educação das crianças (da Infant School ao Parque Infantil, 1823-1935)

Moysés Kuhlmann Jr. ** (iD orcid.org/0000-0001-7564-620X

Para citar este artículo: Kuhlman, M. (2021). El playground y las propuestas para la educación de los niños (desde la Infant School hasta el Parque Infantil, 1823-1935). Revista Colombiana de Educación, l(82) 197-218. https://doi.org/ 10.17227/rce.num82-11383

\begin{tabular}{lr}
\hline (c) $(9)$ & Recebido: $17 / 03 / 2020$ \\
NG & Availado: $22 / 06 / 2020$ \\
$04 / 08 / 2020$
\end{tabular}

* Traducido por Cláudia Schilling, clauschillingl@uol.com.br.

** Doctor en educación. Investigador, Fundação Carlos Chagas. Correo electrónico: mjunior@fcc.org.br 


\section{Resumen}

En la historia del playground desde su desarrollo en EE. Uu. a fines del siglo xIx e inicios del siglo xx, se han adoptado propuestas pedagógicas y de equipos que se refieren a dos instituciones educativas creadas en la primera mitad del siglo xIx, la Infant School inglesa y el kindergarten alemán. La iniciativa norteamericana se extendió en América Latina, como, por ejemplo, en México, Argentina, Uruguay y Brasil. En el artículo se analizan las ideas sobre el playground defendidas por Samuel Wilderspin, responsable del movimiento de las Infant Schools en Inglaterra. Luego, se presentan algunas de las propuestas de Playground Association of America e información sobre la difusión del playground en Latinoamérica. El texto analiza cómo las composiciones que llevaron a su implantación se produjeron a través de articulaciones enfocadas en políticas sociales que no se limitan a cuestiones pedagógicas, escolares, sino que también se refieren a la educación fisica, al urbanismo y al higienismo. Consideradas muchas veces como propuestas restringidas a la educación física, orientadas a las prácticas deportivas, de gimnasia y recreación, estas instituciones componían propuestas de educación integral, involucrando diferentes dimensiones. Se identifican diferencias entre países, que se deben a los contextos en los que se apropiaron las propuestas, como en el caso del Parque Infantil y otras variantes de la institución en Brasil y las Plazas de Deportes y Escuelas al Aire Libre en Uruguay. Las confluencias y distancias en la historia de estas instituciones a lo largo de América Latina constituyen un fértil campo de investigación a explorar.

\section{Palabras clave}

Playground; Infant School educación física; higiene; América Latina

\section{Keywords}

Playground; Infant School; physical education; hygiene; Latin America

\begin{abstract}
In the history of the playground, since its development in the usa, in the late 19th century and early 2Oth century, equipment and pedagogical propositions were adopted which can be traced back to two educational institutions created in the first half of the 19th century: the Infant School, in England, and the Kindergarten, in Germany. The American initiative spread in Latin American countries such as Mexico, Argentina, Uruguay, and Brazil. In this article, we analyze the ideas about the playground advocated by Samuel Wilderspin, who initiated the infant school movement in England. Then, we present some of the propositions of the Playground Association of America and information about how the playground spread in Latin America. The text analyzes how the compositions that led to its implantation were made through social policy arrangements that addressed not only pedagogical and school matters but also physical education, urbanism, and hygiene. Often viewed as exclusively dedicated to physical education propositions -i.e., sports, gymnastics, and leisure practices - these institutions in fact proposed a comprehensive approach to education that encompassed different dimensions. We identify differences between countries which are due to the contexts in which the concepts were appropriated, as with Parque Infantil and other variants of that institution in Brazil and the Plazas de Deportes and Escuelas al Aire Libre in Uruguay The confluences and distances in the history of these institutions across Latin America are a fertile research field to be explored.
\end{abstract}

\section{Resumo}

Na história do playground, desde seu desenvolvimento nos EUA, no final do século XIX e início do século xIx, adotaram-se equipamentos e propostas pedagógicas que remetem a duas instituições educacionais criadas na primeira metade do século xIX, a Infant School inglesa e o Kindergarten alemão. A iniciativa norte-americana espraiou-se na América Latina, como no México, na Argentina, no Uruguai e no Brasil. No artigo, analisam-se as ideias sobre o playground defendidas por Samuel Wilderspin, responsável pelo movimento da Infant School na Inglaterra. Em seguida, apresentam-se algumas das propostas da Playground Association of America e informações sobre a difusão do playground na América Latina. O texto analisa como as composições que levaram à sua implantação foram produzidas por meio de articulações voltadas às políticas sociais que não se restringem a questões pedagógicas, escolares, mas estão referidas também à educação física, ao urbanismo e ao higienismo. Consideradas muitas vezes como restritas a propostas de educação física, voltadas a práticas desportivas, de ginástica e de lazer, essas instituições compuseram propostas de educação integral, envolvendo diferentes dimensões. Identificam-se di-

N. ${ }^{\circ} 82$ ferenças entre os países, que se devem aos contextos em que as propostas foram apropriadas, como no caso do Parque Infantil e outras variantes da instituição no Brasil e das Plazas de Deportes y Escuelas al Aire Libre no Uruguai. As confluências 198 e distâncias na história dessas instituições em toda a América Latina constituem um fértil campo de investigações a ser explorado.

\section{Palavras chave}

Playground; Infant School educação fisica; higiene, América Latina 


\section{Introducción}

Desde los años de 1920, en Brasil, se han desarrollado propuestas y se implantaron instituciones caracterizadas como extraescolares, cuya fuente de inspiración han sido los playgrounds norteamericanos organizados junto a Playground Association of America. Instituciones con perfil similar también han sido identificadas en otros países latinoamericanos, como Argentina, Chile, México y Uruguay. El Parque Infantil, implantado en la ciudad de São Paulo en 1935, fue la institución de mayor notoriedad en Brasil. Desde la década de 1940 han sido inaugurados parques infantiles en el interior del estado de São Paulo, así como en otros estados del país, que se convirtieron, después de la segunda mitad de los años 70, en Escuelas Municipales de Educación Infantil.

En el presente artículo se analizarán las propuestas educacionales para dichas instituciones. Se enfocan el playground de la Infant School inglesa, organizado por Samuel Wilderspin en los años 20 del siglo xix; la Playground Association of America, al inicio del siglo xx, y luego el Parque Infantil y otras instituciones brasileñas y latinoamericanas. Es innegable que cada uno de los contextos en los que se desarrollaron tales instituciones tienen sus especificidades. Sin embargo, también se encuentran puntos comunes en evidencias que remiten a afiliaciones o citas del pasado no explícitas y que se borraron en las narrativas que se ocupan de sus historias. Las composiciones que remiten a su implantación se producen por medio de articulaciones relacionadas con las políticas sociales y no se restringen a temas pedagógicos, escolares, sino que también se refieren a la educación física, al urbanismo, al higienismo. Para desarrollar esa trama surgen como aportes teóricos y metodológicos la perspectiva del estudio de la historia de la educación en el marco de las relaciones sociales y la búsqueda de evidencias que sostienen posibilidades explicativas consistentes (Ginzburg, 2007; Kuhlmann Jr, 2019; Kuhlmann Jr. y Leonardi, 2017; Thompson, 2001; Williams, 1992).

\section{El playground en la Infant School}

Los documentos y los estudios que tratan de la historia del playground mencionan una influencia remota en las propuestas pedagógicas de Froebel para el Kindergarten (Jardín de Infantes). Sin embargo, una figura del libro School architecture, publicado en EE.uv. en 1848, indica otra referencia para la implantación de los playgrounds norteamericanos: la Infant School, institución organizada por Samuel Wilderspin en Inglaterra, que no fue identificada en los textos de la Playground Association of America. El autor del libro, que presentaba diferentes modelos de construcciones 
escolares destinadas a la educación popular, era Henry Barnard, uno de los pioneros del Movimiento de las Escuelas Comunes norteamericano. En un tópico sobre planos para edificios y patios de escuelas infantiles, Barnard reprodujo una figura del playground de la Infant School, argumentando sobre las propuestas de Wilderspin y la importancia de tal espacio, que permitiría alternar horas de recreación y estudio (Barnard, 1849).

En 1823, Wilderspin publicó el libro On the importance of educating the infant children of the poor, en que sistematizó en 184 páginas las propuestas que aplicaba en la Spitalfields Infant School, desde su apertura en 1820, con el propósito de orientar la constitución de un sistema de escuelas infantiles. El libro fue revisto y reescrito algunas veces, recibió el nombre de Early education en 1840 y se publicó en 1852, como octava edición "cuidadosamente revisada", ahora con 351 páginas y denominado The infant system, con el subtítulo "para el desarrollo de las capacidades intelectuales y morales de todos los niños de uno a siete años de edad".

El libro de 1823 ya contiene un capítulo dedicado al playground, que no sería una institución específica, sino un anexo necesario al espacio escolar, al contrario de las instituciones extraescolares implantadas a fines del siglo xIx e inicio del xx, en Estados Unidos. Para el autor, una Infant School sin un playground sería perjudicial a los niños:

\begin{abstract}
Mantener un centenar o más de niños en una sala, aunque pueda ser conveniente en lo que se refiere a otros aspectos, no es adecuado para que los niños relajen y se ejerciten de forma apropiada; ello no podría ocurrir sin un playground, sería como herir materialmente su salud, algo que me parece en mi humilde opinión, de la mayor importancia. (Wilderspin, 1823, p. 170)
\end{abstract}

La misma cita aparece en la edición de 1852, pero en el libro de 1823 las consideraciones del autor son predominantemente morales. El playground podría compararse a estar suelto en el mundo, donde el niño dejaría aflorar sus inclinaciones y los efectos producidos en su educación. En el caso de que tuvieron la tendencia a discutir y pelear, el profesor tendría la oportunidad de aconsejarlos sobre la impropiedad de su conducta, mientras que, si el niño permaneciera tan solo en el aula, ello no se manifestaría en la escuela, sino solamente cuando saliera a la calle. Este espacio para jugar también contribuiría para que al niño le gustara más la escuela. (Wilderspin, 1823, pp. 170-174).

Ya en 1840, los juguetes y juegos ganaron relevancia, al lado de la educación moral. El libro fue citado por Barnard (1849), que reprodujo un grabado que constaba de aquella edición y presentó la organización de aquel espacio externo con materiales y prácticas a desarrollar allí. 


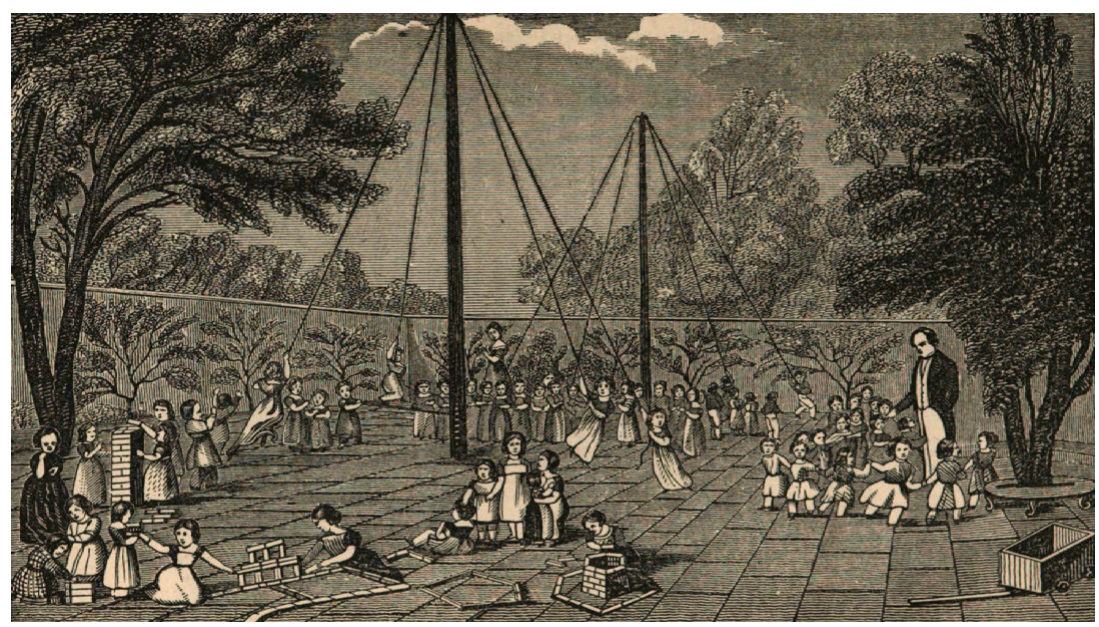

Figura 1. Playground para una escuela infantil o primaria.

Fuente: Barnard, 1849, p. 89.

En la edición de 1852, las propuestas para el playground ya no se restringían a las consideraciones morales y se presentan en dos capítulos: el quinto, sobre los principios para la educación infantil, y el sexto, sobre los requisitos para una escuela infantil.

En el quinto capítulo, el playground surge como algo indispensable a los propósitos educativos de la institución. En la educación infantil, el ejercicio y el fortalecimiento de las energías físicas serían la base para un intelecto poderoso y saludable. Por medio del juego, el niño podría adquirir una gran cantidad de valioso conocimiento. Al jugar, el niño podría ver el libro de la Naturaleza:

La absurda noción de que el niño solamente podría aprender dentro de una sala tiene que acabar. En una hora en los jardines, veredas, campos, sería posible hacer más para cuidar y satisfacer a las florecientes facultades de la niñez que durante meses dentro de una sala de clase. (Wilderspin, 1852, pp. 77-78)

El ejercicio físico, inherente a la vida animal, sostenía la propuesta de que una buena constitución debería ser el primer objetivo de la educación. La adquisición de hábitos de trabajo no se podría hacer por medio de la restricción de las actividades infantiles:

Quiten a los niños su entretenimiento y pronto dejarán de ser los vivos y felices seres que hemos visto hasta ahora, y se convertirán en criaturas enfermizas y sin vida que estamos acostumbrados a ver y lamentar, sujetos al confinamiento y la restricción de las dame schools. No tengo escrúpulos en afirmar que, si se retiraran los playgrounds de las escuelas infantiles del sistema, a partir de este momento los niños dejarían de ser una bendición para el país. (Wilderspin, 1852, p. 80) 
O sexto capítulo prevé que los docentes vivan en la escuela y dispone sobre materiales, muebles, arreglo de los espacios y distribución de los niños por edad. En él, Wilderspin no reproduce la figura de la edición de 1840, sino que presenta una representación más simple del columpio giratorio (rotatory swing) existente en el playground, que también es la imagen de la portada de la publicación. Después de mencionar las consideraciones de orden moral ya previstas en 1823, el autor se extiende sobre la organización del espacio. Recomienda la pavimentación del piso con ladrillos, a fin de obtener un buen drenaje del suelo y evitar que los niños se ensucien demasiado. Habría que plantar árboles fructíferos alrededor de los muros y al centro del playground, lo que alegraría a los niños, enseñándoles también a respetar la propriedad privada. También se recomienda un cantero de flores alrededor del playground. Todo ello proporcionaría una oportunidad de lecciones útiles para los niños.

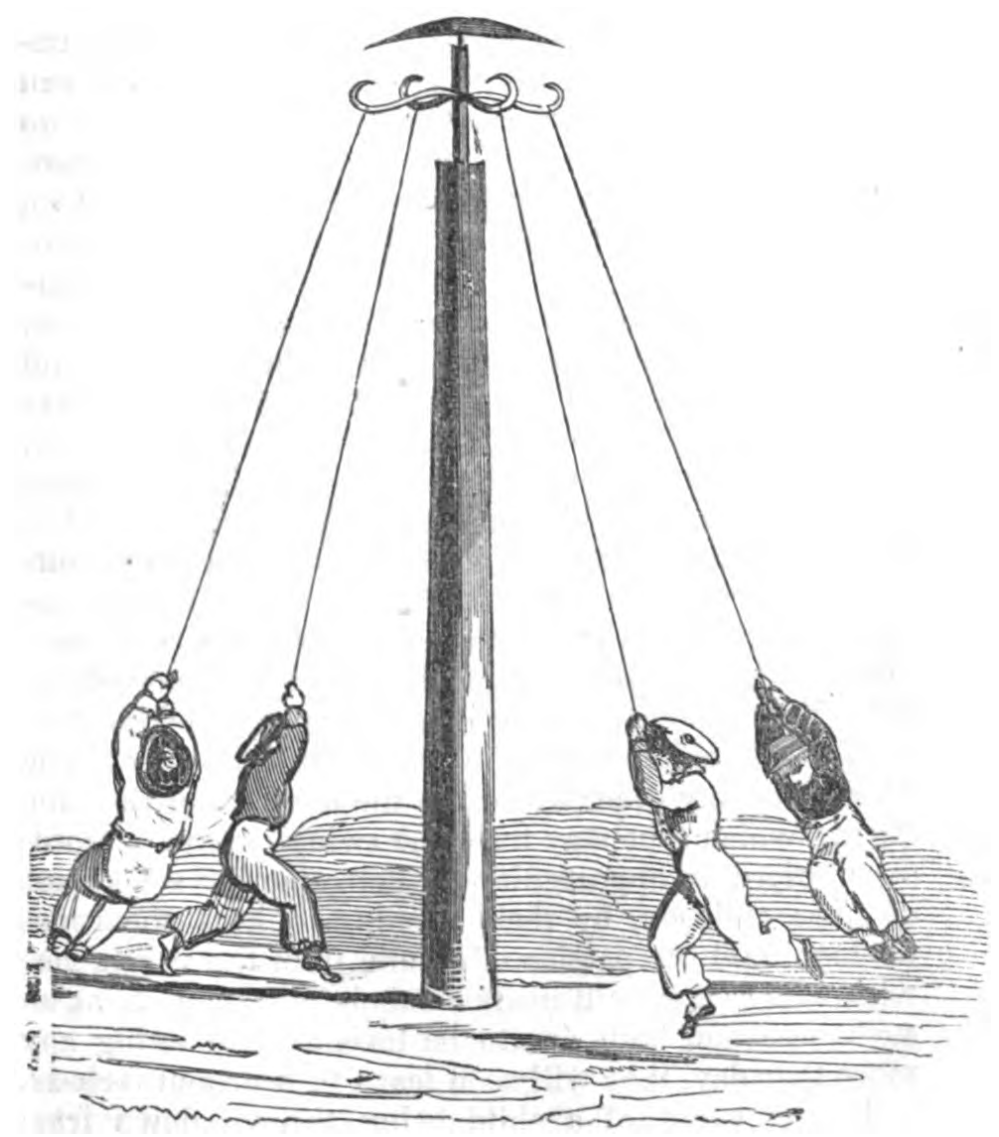

Figura 2. columpio giratório.

Fuente: Wilderspin, 1852, p. 105. 
Asimismo, Wilderspin manifestó su creciente convicción en la importancia del playground a cada año. Relató que la experiencia con el uso de pelotas, aros para girar y plumillas no había sido exitosa, ya que las pelotas se perdían a menudo al sobrepasar los muros, y los otros materiales provocaban accidentes. Surgió entonces la idea de obtener bloques de madera con cerca de 10 centímetros de largo por 5 de ancho y 3 de altura, con los cuales los niños mucho se entretenían, construyendo una gran variedad de formas. (Wilderspin, 1852, pp. 101-105)

Para completar el playground, Wilderspin proponía que se instalara un columpio giratorio con un mástil de cerca de 5 metros de altura, en cuyo tope se instalarían 4 cuerdas en una roldana para que los niños giraran, un ejercicio que fortalecería sus músculos y cuerpo. El autor finalizó el capítulo afirmando incluso que el playground podría recibir el nombre más apropiado de training-ground, campo de entrenamiento (Wilderspin, 1852, pp. 105-107). El columpio giratorio fue un equipo utilizado en los playgrounds y en las instituciones brasileñas, denominado "paso del gigante" (Kuhlmann Jr., 2019).

Los bloques en madera, como representado en la figura de 1840, son muy similares a los materiales utilizados en el jardín de infantes. Aunque el uso de dichos materiales no haya ocupado un lugar tan central en la Infant School como en la propuesta froebeliana, los indicios muestran que el uso de tales materiales de construcción no fue una idea original del pedagogo alemán. Es viable suponer que en ambos casos serían apropiaciones de prácticas oriundas de juegos de la cultura popular. No se sabe si Froebel leyó el libro de Wilderspin, lo que no es improbable, ya que fue traducido al alemán por Joseph Wertheimer en 1826, contando con una segunda edición en 1828, con 410 páginas (Wilderspin, 1828). Según Jean-Noel Luc (1999, pp. 192-193), Wertheimer impulsó el debate sobre la educación de los niños en Hungría e Italia, además de Alemania.

\section{El playground en Estados Unidos}

En lo que se refiere al playground norteamericano presentamos algunas informaciones. Las ideas de Wilderspin no fueron referidas por los protagonistas del movimiento. Sin embargo, la figura publicada en el libro de Barnard fue apropiada en la historiografía de los playgrounds y difundida equivocadamente como se fuese una propuesta elaborada por el propio Barnard o incluso como una imagen de lo que habría sido uno de los primeros playgrounds norteamericanos (Brett, Moore, Provenzo, 1999; Kuhlmann Jr., 2019).

La referencia a Froebel aparece como fuente de inspiración para las propuestas, como se refiere en el primer número de la revista mensual The Playground, de Playground Association of America, en abril de 1907: 
Froebel planificó el kindergarten hace más de ochenta años, con sus juegos y ocupaciones para niños pequeños. Ahora proponemos desarroIlar un sistema graduado de kinder-welten, - escuelas con playgrounds y talleres, gimnasios y salas de trabajos manuales, salas de ciencia y arte, museos y bibliotecas, salas de lectura y de estudios, con un currículo que presente las sucesivas fases del progreso humano, a fin de que el niño individual y su medio puedan actuar y reaccionar en líneas seleccionadas de desarrollo racial. (The Playground, 1907, p. 8)

La revista, lanzada un año después de la creación de la Asociación, traía una carta del presidente Theodore Roosevelt en defensa de los playgrounds públicos. Roosevelt fue nombrado presidente de honor de la entidad, que también indicó como miembro honorario el Barón Von Schenkendorff, presidente de la Asociación de los Playgrounds de Alemania.

Von Schenkendorff fue considerado como referencia para la organización de los playgrounds en EE.uU., que habrían sido implantados en función de la visita de Marie Zakrzewska a Berlín, cuando observó jardines con arena para los niños creados por él, y que inspiraron la implantación de un amplio tanque de arena en los jardines de la Children's Mission en Boston, en 1885 (Hansan 1925).

En el cuarto número de la revista The Playground, se desarrolla la idea del kinderwelten, el mundo de los niños, que ampliaría la idea de Froebel, con las escuelas de juegos (play schools). Las escuelas de juego congregarían playgrounds, haciendas de juegos (play farms), escuelas de vacaciones y sus tiendas:

Las escuelas de juegos son necesarias para el desarrollo de la expresión, poder, personalidad - mitad del trabajo de la educación pública fundamental, ignorada o poco desarrollada en el actual sistema de educación, que limita su atención mayoritariamente al trabajo de pensamiento, refinamiento, cultura (Stewart, 1907, p. 7).

El folklore fue uno de los recursos recomendados por la Playground Association of America. En la programación de su Primer Congreso Anual, en 1907, se preveía la presentación de danzas folklóricas de Polonia, Bohemia, Italia, Grecia y Noruega (The Playground, n.3, p.8). En el Segundo Congreso Anual de Playgrounds, que se Ilevó a cabo en 1908, en Nueva York, el festival de danzas nacionales y folklóricas fue considerado el más bello evento de la reunión. Las danzas folklóricas presentadas por los niños provenían de varias nacionalidades: italiana, polonesa, española, irlandesa, bohemia, rusa, sueca, húngara, escocesa, alemana y "negro" (sic) (Playground Association, 1909, pp. 48-49).

La danza folklórica proporcionaría situaciones controladas en las que el reconocimiento de las diferencias culturales de la población inmigrante representaría un medio para su integración a la sociedad norteamericana. 
Al sentir que sus herencias culturales eran apreciadas, los recién Ilegados podrían desarrollar más fácilmente la lealtad a la nueva nación. Al bailar en conjunto, crecerían menos apegados a sus diferencias y más conscientes de su esfuerzo individual como subordinados a los intereses del grupo (Mooney-Melvin, 1983).

Luther Halsey Gulick, presidente de PAA, pensaba que no bastaría considerar los juegos y danzas como una válvula de seguridad, como un valor moral, que ofrecerían la oportunidad para un inocente consumo de alegre energía:

Creemos que ellos constituyen una fuerza moral positiva, una agencia social, que tuvieron en el pasado y están destinados a tener en el futuro la gran función de moldear en un todo único aquellos cuyas condiciones y ocupaciones son demasiado diversas (1909, p. 433).

La asociación tuvo su nombre alterado para Playground and Recreation Association of America a mediados de la década de 1910, y para National Recreation Association en los años de 1930. La retirada del nombre Playground habría representado el final de la defensa de los playgrounds organizados para los niños, con la transición hacía el énfasis en el playground como un espacio de ejercicios de gimnasia o de recreación en las escuelas o parques públicos, aunque la propuesta original haya permanecido en el interior de las escuelas de educación infantil (Frost y Woods, 1998).

\section{La educación física y el playground en países de América Latina}

Hay varios indicios de la inspiración norteamericana en lo que se refiere a la instalación de instituciones relativas a los playgrounds en los países de Latinoamérica, fuertemente vinculadas a la educación física.

En Brasil, Frederico Gaelzer, atleta de la Asociación Cristiana de Jóvenes en Porto Alegre, Rio Grande do Sul, fue becado para estudiar en Estados Unidos, en el George Williams College, institución vinculada a la YMCA, en 1918. Regresó a Porto Alegre solamente en 1926, donde organizó, junto con la alcaldía, los Jardines de Recreación, versión regional de los playgrounds (Feix y Goellner, 2008). Según Gomes (2006, p.105-106), antes de su retorno, actuó en las ACJ de México y Uruguay.

La denominación Jardín de Recreación compone las referencias a Froebel y al playground. En el caso brasileño se utilizaron otras denominaciones, como Campo o Plaza de Recreación y de Juegos, Escuela de Salud y Parque Infantil, que se convirtió en la más común. 
La denominación parque infantil ya aparece en 1915, en el libro Eduquemos, de Arthur Porchat de Assis, profesor de Pedagogía en el Liceo Femenino Santista y director del Instituto Dona Escholástica Rosa, en Santos, São Paulo (Faria y Monteiro, 2020). En el libro, el autor concluye el capítulo titulado "De la Educación Physica" proponiendo la creación de parques infantiles. Assis cita a Froebel, pero no está claro si hay influencia del modelo de los playgrounds, incluso porque la principal referencia del autor en el libro es europea y no norteamericana. Pero las proposiciones se acercan a lo que después se configuraría en la implantación de tales instituciones en Brasil:

En lo que se refiere a la iniciativa privada, anímense los propietarios de los casinos de nuestras playas a establecer los grandes parques infantiles, donde los niños puedan disfrutar con la oxidación del aire, la libertad de sus movimientos, la alegría y expansión de sus sentimientos. ¡Nada podrá ser más atractivo que un parque infantil! Por la mañana, suelten las familias ese grupo parlanchín de niños, y que vayan a buscar en el parque los más inocentes motivos para intercambiar ideas, afectos, corriendo por las extensas alamedas, a pie, en sus bicicletas, en sus carritos tirados por chivos; que vayan a construir sus casitas de arena al lado de la laguna cristalina; que edifiquen artísticos palacios, armados con cubos y otros materiales de madera del Papá Froebel; en suma, todo que exprese vida, salud y alegría. Los niños pasarán horas desapercibidos, disfrutando saludablemente las influencias de ese medio real y artificial. Real, por la aproximación directa de la naturaleza, cuyos elementos físicos exteriores actuarán, restaurando, en su organismo general; artificial, por los efectos educativos que el arte crea en la elección de los juegos, en la variedad de las ocupaciones, siempre improvisadas y accionadas por los directores de esos centros infantiles. (Assis, 1915, pp. 28-29)

En 1941, Nicanor Miranda, que era jefe de la División de Educación y Recreación de São Paulo, responsable por los parques infantiles del municipio, publicó un libro sobre la propagación de los parques infantiles y parques de jugos. En relación a América Latina, Miranda presentó informaciones sobre iniciativas en México, Cuba, Uruguay, Argentina y Chile. Sobre México, indicó que los parques representaban un desdoblamiento de las "misiones culturales" desde 1926, y destacó el papel de los profesores de educación física en la recuperación de los juegos y danzas tradicionales mexicanos, realizados en el parque infantil de Xalapa. En Cuba, mencionó la creación del Consejo Corporativo de Educación, Salud y Beneficiencia, en 1936, en el gobierno de Batista, responsable por la apertura de parques infantiles y juveniles, destacándose el parque José Martí, en La Habana. En Uruguay, Miranda afirmó que la educación física era estimulada desde 1923, con las "plazas de deportes", que tradujo parques de juegos, algunas 
de ellas con un "rincón infantil". En lo que concierne a Argentina, se refirió a la creación de la "Dirección de Plazas de Ejercicios Físicos y su Reglamentación", en 1919, que organizó plazas de juegos, colonias de vacaciones, clubes de chicos jardineros y recreaciones infantiles. En lo que se refiere a Chile, mencionó la creación de plazas de juegos desde 1923; muchas de ellas fueron cerradas porque no se habían implantado servicios organizados y con profesionales, restringiéndose muchas veces a una simple instalación de equipos (Miranda, 1941, pp.15-19).

Sin embargo, hay que observar con cautela los documentos. Las informaciones de Miranda se obtuvieron a través de una bibliografía a la que accedió y que contiene incorreciones. Por ejemplo, en relación con Uruguay, Miranda se refiere al documento de autoría de Julio Rodríguez, Plan de acción de la Comisión Nacional de Educación Física (CNEF) de 1923. Pero las iniciativas en Uruguay venían del año de 1911, en el que se llevó cabo la creación de la comisión en que Rodríguez posteriormente actuó como director técnico. En 1913 ocurrió la implantación de las Plazas Vecinales de Cultura Física, cuyo nombre fue modificado para Plazas de Deportes en 1915 (Scarlato, 2015). Es posible que Miranda se haya equivocado o que haya ocurrido un error gráfico en su trabajo, ya que presenta como referencia el informe de 1923 y afirma en el texto que sus informaciones sobre Uruguay provinieron del "último informe oficial" de aquel país, en el que ya existirían 85 plazas de deportes (Miranda, 1941).

Sobre Uruguay, Scarlato (2015, pp. 111-112) menciona el libro de Juan Arturo Smith, presidente de CNEF, publicado en 1913, que rendía tributo a Estados Unidos, refiriéndose al libro American playgrounds, de E. B. Mero, de 1908. Miranda (1941) también mencionó este libro como referencia para escribir sobre EE.uu. Según Scarlato, Smith consideró que las plazas vecinales superaban los playgrounds por dos motivos: no estaban abiertos solamente a niños y adolescentes, sino a las madres, padres, jóvenes y adultos mayores; y por la incorporación de una "dirección organizada". Smith nombró a Jess Hopkins como director técnico de la primera plaza vecinal. Hopkins era un estadunidense radicado en Uruguay desde 1912, graduado en educación física en el Springfield College, vinculado a la YMCA, que se convirtió en el encargado del Departamento de Educación Física de la ACJ, Asociación Cristiana de Jóvenes uruguaya. Posteriormente, por indicación de Hopkins, Julio Rodríguez fue a estudiar educación física en la Universidad de Springfield.

Cabe señalar que el presidente de la Playground Association of America, Luther Halsey Gulick, también fue director del Departamento de Educación Física de la Universidad de Springfield, anteriormente denominada Escuela Internacional de Capacitación de la YMCA, en Massachussets (Dogliotti, 2013, p. 2). 


\section{El urbanismo y los playgrounds}

El crecimiento de las ciudades, a fines del siglo xix e inicio del siglo xx, trajo el tema de los parques y la recreación a los debates del urbanismo y del paisajismo. En 1923, Gilberto Freyre, después de concluir su maestría en la Universidad de Columbia, escribió la crónica Ludum Pueris Dare, publicada en el Diário de Pernambuco, en el que mencionaba los playgrounds:

Quisiera que los niños mis compatriotas supieran resistir a la manía que existe aquí, la de convertir a los niños en hombrecitos lo más pronto posible. Ya basta el hecho de ser este burgo, como los demás burgos de Brasil, una triste ciudad sin áreas de recreación para los chicos, sin céspedes por los que puedan correr, sin tanques donde puedan jugar con navíos de papel, sin nada que les estimule la alegría. Y, al contrario de los chicos que vemos, en revuelos alegres, por la hierba de los parques de Londres y Berlín, en las Tullerías y en los "playgrounds" de cualquier ciudad de Estados Unidos y Canadá, acá los niños son tristes criaturas, candidatos al traje de etiqueta y a la calvicie precoz. (1979, p. 141)

En 1925, Gilberto Freyre volvió a mencionar en otra crónica la necesidad de áreas para la libre recreación infantil y en 1929, cuando fue secretario del gobernador de Pernambuco y profesor de sociología en la Escuela Normal de Recife, hizo algunos apuntes sobre investigaciones realizadas por sus alumnas en la ciudad y la inminencia de implantar playgrounds. Freyre había efectuado estudios sobre el urbanismo moderno y proyectaba lo siguiente: "Recife será la primera ciudad brasileña a tener playgrounds". No se sabe si con efecto han sido implantados, ya que con los cambios políticos que ocurrieron en Brasil en 1930, el gobernador fue destituido y Freyre lo acompañó al exterior (Freyre, 2006, p.314).

En 1930, en la ciudad de São Paulo, se inauguró el Playground del Parque D. Pedro II, resultado de articulaciones realizadas desde la década de 1920 en el ámbito del Rotary Club, presidido por el médico Edmundo de Carvalho, del que participaba el futuro alcalde de la ciudad, Luiz Ignácio de Anhaia Mello (Dalben, 2016). Para los urbanistas, la recreación activa de los niños propiciaría el contacto con la naturaleza. Soares analiza cómo, al inicio del siglo $x x$,

educadores, científicos, artistas, urbanistas y médicos contribuyen para configurar un ideario de vida al aire libre, en el que es central el deseo de evadirse rumbo a una naturaleza concebida como fuente de recuperación de energías perdidas y de regeneración. (2016, p. 18)

No se trata tan solo de una naturaleza externa al ambiente urbano, sino también del desarrollo de propuestas de la organización de espacios, que traerían la naturaleza hacia el interior de la ciudad. Las ideas urbanistas estuvieron presentes en el playground del Parque D. Pedro II, en São Paulo, 
inaugurado el 25 de diciembre de 1930. En Navidad hubo una fiesta con la distribución de juguetes y golosinas para los niños y que contó con la presencia de autoridades.

Es probable que el playground hubiera comenzado a funcionar anteriormente, siendo esta fecha tan solo para la conmemoración oficial, ya que 7 meses antes un reportaje del Diário Nacional anunciaba que las obras ya se encontraban en fase de conclusión. El punto de partida del texto eran las concepciones del "urbanismo moderno" en relación al restablecimiento del contacto con la naturaleza, indicando la tendencia de traer el campo a la ciudad por medio de la implantación de la "recreación activa". Con una pileta para niños de hasta 5 años ya pronta, el primer parque de recreación organizado de la ciudad aguardaba los últimos retoques en su galpón y la entrega de los sube y bajas, hamacas y otros juegos que eran construidos en el Liceo de Artes y Oficios de la capital. Se preveía la asistencia diaria de 300 niños. Considerando el urbanismo como una cuestión relativa a la educación, el reportaje presentaba la finalidad educativa del playground:

al lado del perfeccionamiento físico de los niños se planteará su formación mental, por medio de discursos educativos, clases al aire libre, de un cuarto de hora, canciones, poemas etc. [...] Los niños respirarán la atmósfera libre del campo en plena ciudad de los rascacielos. ("Están casi listas", 1930)

\section{El higienismo - educación y salud en los parques}

En 1931, la denominación del playground del Parque D. Pedro ॥ fue alterada para Escuela de Salud, resultado de una alianza de la Asociación Cruzada Pro-Infancia con la alcaldía de São Paulo, cuya base era un programa destinado a la niñez que tenía el propósito de reducir la mortalidad infantil y promover la educación para la salud física y moral de los niños. La autora del programa fue Maria Antonieta de Castro, directora secretaria de la entidad, cuya directora general era Pérola Byington. Maria Antonieta de Castro era educadora sanitaria y había ejercido varios cargos en el Servicio Sanitario y en el Instituto de Higiene de São Paulo. La propia Cruzada Pro-Infancia nació en el interior de la Asociación de Educación Sanitaria, y se convirtió en un ente autónomo en 1931.

El 12 de octubre de 1931 se llevó a cabo la inauguración oficial de la Escuela de Salud, como parte de las conmemoraciones de la Semana del Niño, festividad implementada por la Cruzada Pro-Infancia. Durante la ceremonia, Pérola Byington, directora de la Cruzada, expuso que la finalidad de la escuela era atraer a los niños de los barrios cercanos, como Brás y Moóca, que en la época eran sitios en los que vivían los obreros. 
En su opinión, el playground del parque D.Pedro II estaba prácticamente abandonado, contando solamente con vigilancia diaria, pero sin orientaciones. La alcaldía atendió la solicitud de la Cruzada y le cedió el espacio, que pasaría a contar con un programa educativo, con conferencias, reuniones de padres, gimnasia, juegos, juguetes, excursiones educativas, gimnasia médica especializada, fiestas y competencias deportivas. En la tarde de ese mismo día, Pérola Byington y Maria Antonieta de Castro participaron en la continuación de las conmemoraciones de la Semana del Niño, en el Teatro Municipal, donde se realizó la conferencia de Fernando de Azevedo, "La Salud y la Escuela Nova". En la apertura de la sesión, el Director General de Enseñanza de São Paulo, Lourenço Filho, leyó una carta del presidente de la República, Getúlio Vargas, en la que manifestaba su interés por la Semana del Niño promovida por la Cruzada y por una campaña que se ocupaba de los problemas relativos al niño en la prensa, estaciones de radio y conferencias. Fernando de Azevedo, en su discurso, mencionó la necesidad de proteger la salud del niño, además de formar su carácter y educar su inteligencia. ("Tuvo inicio ayer", 1931)

Sobre la propuesta educacional de la Escuela de Salud, que contaba con educadoras sanitarias comisionadas, Maria Antonieta de Castro escribió lo que sigue:

En 1931, la Cruzada organizó y puso en funcionamiento, en el Parque D. Pedro, la Escuela de Salud para personas con debilidades físicas, con un régimen especial de ejercicios físicos, hidro y helioterapia, en la que también organizó la primera Biblioteca Infantil de S. Paulo, con 500 volúmenes. En 1936, asimilada por la Alcaldía, esta Escuela se transformó en el Parque Infantil que todavía existe en la actualidad. (Castro, 1956, p. 2)

La ciudad de Santos, en el litoral de São Paulo, también inauguró su Escuela de Salud, bajo la dirección del Rotary Club de la ciudad, incluso antes que la de São Paulo, el día 23 de febrero de 1931. Posteriormente, en 1942, se incorporó a la alcaldía de Santos, con la denominación de Parque Infantil (Cunha, 2018).

De la misma forma que en la ciudad de São Paulo, se preveía que la Escuela de Salud tuviese educadoras sanitarias vinculadas al estado, auxiliadas por adjuntas municipales, que impartirían "enseñanzas de gimnasia respiratoria y sueca, recreación instructiva, instrucción cívica y nociones de cosas e higiene, además de la práctica de la helioterapia" (Escuela de personas con debilidades, 1931). 
La expresión Escuela de personas con debilidades, que era el título de la nota, también se utilizó en relación con la Escuela de Salud de la ciudad de São Paulo y remite a la propuesta de las escuelas al aire libre y de las colonias de vacaciones, difundidas desde el inicio del siglo xx, en Europa y América Latina (Amaral 2016, Dalben 2009, 2019). Serían medidas destinadas a superar la debilidad física y el riesgo del contagio de la tuberculosis, en épocas anteriores a la vacuna BcG.

El sol y la naturaleza como promotores de la salud fueron propagados por los higienistas. En 1916, Moncorvo Filho, director del Instituto de Protección y Asistencia a la Infancia de Rio de Janeiro, presentó una comunicación al Primer Congreso Médico Paulista sobre las iniciativas brasileñas en lo que se refiere a la helioterapia. Además de su actuación, destacó los nombres de "Clemente Ferreira, Alfredo Ferreira de Magalhães, Augusto Paulino, V. Veiga, Jader de Azevedo, Ribeiro de Castro, Oliveira Botelho, Julio Novaes y otros", que hacía tres años comenzaban a ensayar el "nuevo método naturista" (Moncorvo Fo 1917, p.8). En el texto, se refirió a la creación de un Servicio especial de helioterapia, con la instalación de un solárium en el Dispensario Moncorvo, presentando resultados de 14 casos de tratamiento de niños y adolescentes.

El 04 de mayo de 1924, Moncorvo Filho, juntamente con Alves Filgueiras, inauguró el "Heliotherapium", "un establecimiento especialmente consagrado a la profilaxis y cura de las enfermedades por medio de los baños de sol". En el discurso pronunciado en la inauguración, Moncorvo afirmó que uno de los principales objetivos del instituto se resumiría a:

cuidar particularmente de los niños débiles, apocados, anémicos o raquíticos, cuyo físico sería radicalmente transformado por los maravillosos efectos de la vida al aire libre, aprendiendo o jugando bajo el influjo de los rayos metódica y escrupulosamente empleados (Moncorvo Fo 1924, pp. 6-7).

En lo que concierne a la helioterapia, las imágenes de los niños en fotografías de la Escuela de Salud de Santos siguen el patrón de escuelas al aire libre europeas o de otros países latinoamericanos. Los niños usan sombreros de ala ancha con ropas de baño. En una de las fotos, están en formación y está escrito en la foto, "gimnasia al sol"; en otra, un modelo clásico: la mayoría de los niños está acostada en sillitas de lona, dispuestas en semicírculo, además de otros sobre esteras en el piso, y frente a ellos se encuentra la profesora, vestida, sentada en un banquito, con la frase "baños de sol con lecciones de higiene" (Cunha 2018, p. 64). 


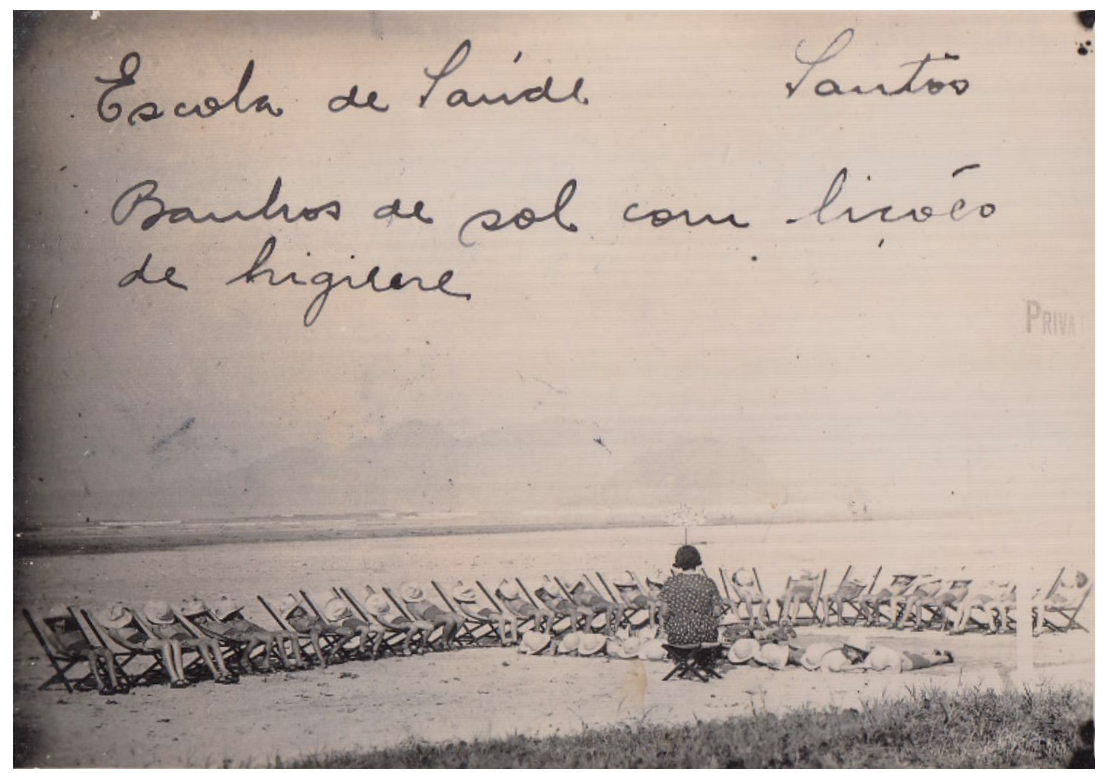

Figura 3. Baño de sol en la Escuela de Salud de Santos, s. d.

Fuente: Acervo familia Duarte. Laboratorio de Información, Archivo y Memoria de la Educación (LIAME), Unisantos.

La denominación Escuela de Salud ya se había utilizado anteriormente a la institución de Santos y al playground del Parque D. Pedro II. En julio de 1930, un reportaje se ocupaba de la Escuela de Salud del Centro de Salud de Bom Retiro, barrio de la capital de São Paulo, instalada hacía dos meses por el Servicio de Inspección Sanitaria del estado. La encargada del Centro, Maria Conceição Junqueira, relató a los periodistas que la intención era convertir niños débiles en niños robustos. Para ello, seleccionaron 20 chicas y 15 chicos desnutridos, del Grupo Escolar João Kopke, cerca del Centro de Salud. Pasaron entonces a trasmitirles enseñanzas sobre la salud, a administrarles medicamentos, gimnasia respiratoria y exposición al sol. Al final de las actividades servían una sopa o una papilla y una fruta. Las educadoras sanitarias también visitaban a los padres para aconsejarlos. Como estrategia didáctica, elaboraron excursiones denominadas "rumbo a la ciudad de la salud". Según la propuesta, para llegar a la ciudad de la salud, los niños tendrían que pasar por todas las estaciones, lo que significaría ganar 200 gramos de peso, mensualmente:

como en los cuentos de hadas, la Ciudad del Baño es la primera estación de la carretera de la higiene, que Ilegará a la Ciudad de la Salud, pasando por las estaciones: Fuente de Agua Pura, Valle de las Frutas, Ciudad de la Alimentación Saludable, Ejercicio y Reposo, Aire Puro y Luz del Sol, Estancia de la Leche, Campo de las Papas, Escuela, Sueño reparador. ("Apenas de tanga", 1930) 


\section{Consideraciones finales}

Las relaciones entre las propuestas para el playground de la Infant School y el playground norteamericano no fueron directas, pero hay interesantes puntos de contacto que repercutieran en la difusión de esta institución por el continente americano. El columpio giratorio (rotatory swing), cuyo proyecto se presentó en detalle en el libro de Wilderspin, acompañó dicha difusión, denominado "paso del gigante" en Brasil, siendo el precursor de otros equipamientos (toboganes, sube y bajas, hamacas, carruseles) que pasaron a ser instalados tanto en playgrounds institucionales - organizados, con un cuerpo profesional -, como en playgrounds más informales - un espacio en plazas y parques para la diversión de los niños. No se localizó ningún estudio que tratase de la repercusión del libro de Barnard sobre la arquitectura escolar en Estados Unidos. Sin embargo, la apropiación de la imagen de Wilderspin como si fuera el proyecto precursor de un playground norteamericano es un reconocimiento distorsionado de tales relaciones.

La educación física se convirtió en una de las principales bases para propagar esa institución educacional. El cuerpo se pasó a comprender más allá de la práctica de ejercicios de gimnasia, en las expresiones culturales, como en el caso de las manifestaciones folklóricas, y en su relación con la naturaleza, promotora del desarrollo saludable.

La formulación de políticas sociales al lado de la planificación de las ciudades modernas y sus espacios agregan el interés de urbanistas y gobernantes sobre la institución. El presidente norteamericano Theodore Roosevelt, en su carta a la Playground Association of América escribió lo que sigue:

El juego es en la actualidad prácticamente el único método para el desarrollo físico de los niños de la ciudad y tendríamos que proveer instalaciones para ello, si deseamos niños fuertes y cumplidores de la ley. [...] Si no permitimos que los niños trabajen, tenemos que garantizarles otros espacios que no sean las calles para su tiempo de recreación. (The Playground, 1907, p. 5)

Los vínculos entre educación y salud representaron un fuerte componente de las propuestas de tales instituciones, estando presentes incluso en un periodo posterior al indicado en el título de este artículo. Curiosamente, la actividad de la excursión desarrollada en la Escuela de Salud de Bom Retiro fue propuesta de forma muy similar en junio de 1948.

Entre 1947 y 1957, las propuestas para el Parque Infantil en la ciudad de São Paulo se difundían en el Boletim Interno de la División de Educación, Asistencia y Recreación de la Secretaría de Educación de la ciudad de São Paulo, que mensualmente orientaba el trabajo en aquellas instituciones (Kuhlmann Jr y Fernandes, 2014). En el Boletim Interno publicado aquel 
mes, la directora de la Sección Técnico-Educacional, Noemia Ippolito, presentaba una reproducción en miniatura de un dibujo existente en el edificio del Parque Infantil D. Pedro II, con el nombre de "Un viaje a la tierra de la salud" (fig. 4). El dibujo se debería entregar a los niños, que con su atracción por la manipulación de los lápices de color pintarían las imágenes, "simulando un verdadero viaje" y adquiriendo conocimientos indispensables sobre el baño, la vida al aire libre, la alimentación saludable, pero también sobre las instituciones educativas y el estudio. Habrá sido la propuesta de Bom Retiro utilizada como referencia para la confección del dibujo todavía en la época de la Escuela de Salud en el Parque D. Pedro II, ¿y se habrá mantenido después de la mudanza al Parque Infantil?

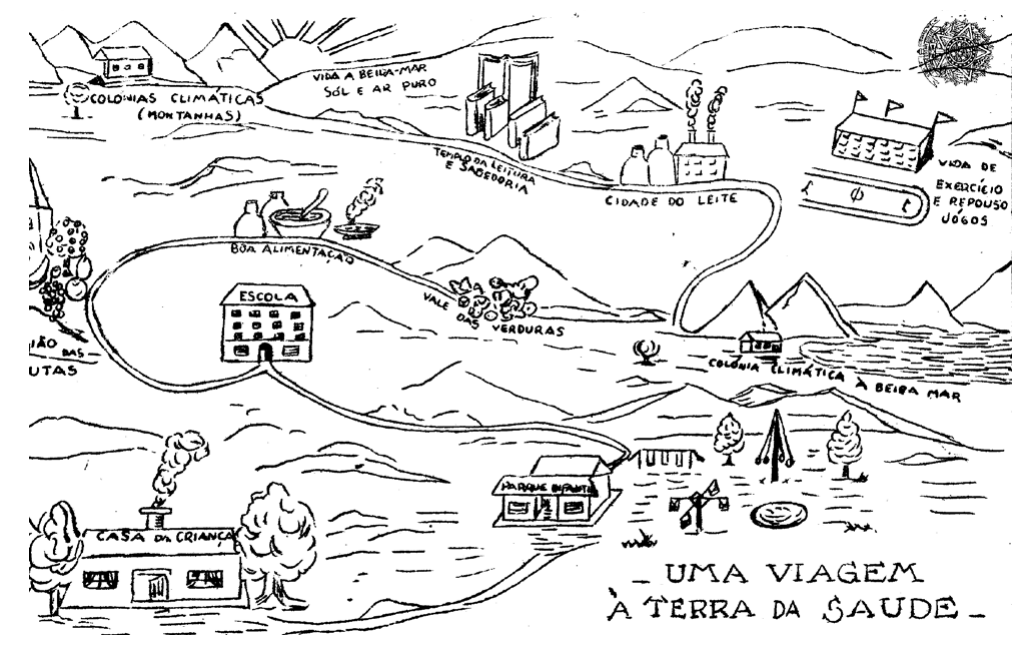

Figure 4. Educação Sanitária.

Fuente: Boletim Interno da Divisão de Educação, Assistência e Recreio (1948, jun.)

En las Escuelas de Salud y en el Parque Infantil instalados en São Paulo las orientaciones relacionadas al ámbito de la educación física, desde la perspectiva de la educación integral, se integraron a las del higienismo, desde la perspectiva de las escuelas al aire libre. Sin embargo, en Uruguay, según lo que todo indica, las vertientes de la educación física y del higienismo siguieron caminos paralelos. Las Plazas de Deportes recibían niños y jóvenes para ejercicios y juegos recreativos y la práctica de deportes, como el fútbol, voleibol, tenis y boxeo (Scarlatto, 2015). Al mismo tiempo se inauguraban en 1913 las Escuelas al aire libre, por iniciativa de la Liga Uruguaya contra la Tuberculosis, contando con la colaboración del Cuerpo Médico Escolar y la Dirección General de Instrucción Primaria (Dalben, 2019).

Las confluencias y distancias en la historia de dichas instituciones en toda América Latina constituyen un fértil campo de investigaciones a explorar. 


\section{Referencias}

Amaral. A. (2016). Aprender a palavra ao ar livre. Estratégias de modernidade sanitária em Portugal no início do séc. xx. História. Revista da FLUP. Porto, 6, 41-55. https://ojs.letras.up.pt/index.php/historia/ article/view/1716/1524

Assis, A. (1915). Eduquemos. Instituto D. Escholastica Rosa.

Barnard, H. (1849). School architecture; or contributions for the improvement of school-houses in the United States. A. S. Barnes. https:// books.google.com.br/books?id=7wwyAQAAMAAJ\&printsec=frontcover \&hl=pt-PT\&source=gbs_ge_summary_r\&cad=0\#v=onepage \&q\&f=false.

Brett, A., Moore, R. y Provenzo, E. (1993). The complete playground book. Syracuse University Press.

Castro, M. A. (1956). A Cruzada Pró Infância: finalidades, organização, realizações. Relatório apresentado pela Diretora Secretária em 12 de Agosto de 1956.

Cunha, H. P. (2018). De Escola de Saúde a Parque Infantil: Santos (19311952). [Tesis de maestria, Unisantos]. Repositorio institucional Unisantos https://tede.unisantos.br/handle/tede/4801

Dalben, A. (2009). Educação do corpo e vida ao ar livre: natureza e educação física em São Paulo (1930 - 1945). [Tesis de maestria, Unicamp]. Repositorio institucional Unicamp http://repositorio.unicamp.br/jspui/ handle/REPOSIP/274792

Dalben, A. (2016). Notas sobre a cidade de São Paulo e a natureza de seus parques urbanos. Urbana: Revista Eletrônica do Centro Interdisciplinar de Estudos sobre a Cidade, 8(2) 3-27. https://doi.org/10.20396/ urbana.v8i2.8643241

Dalben, A. (2019). Las escuelas al aire libre uruguayas: creación y circulación de saberes. Educación Física y Ciencia, 21(2). https://doi.org/10.24215/23142561e075

Diário Nacional. (1930, 9 de mayo). Estão quasi promptas as obras do "playground" do Parque D. Pedro II.

Diário Nacional. (1930, 23 de julio). Apenas de tanga, as crianças recebem em cheio os raios.

Diário Nacional. (1930, 25 de diciembre). Inaugura-se hoje, ás 10 horas, o "play-ground" do parque D. Pedro ॥.

Diário Nacional. (1931, 11 de enero). Escola de débeis.

Diário Nacional. (1931, 13 de octubre). Iniciou-se ontem a semana da criança.

Dogliotti Moro, P. (2013) Imágenes del "maestro de cultura física" en la revista Uruguay-Sport: archivos de la cNEF. Imagens da Educação, 3(1), 1-10. 
Faria, L. H. P. y Monteiro, T. D. B. C. (2020). Arthur Porchat De Assis e o discurso da modernidade educacional em seu livro "Eduquemos" (1915). xIv congresso iberoamericano de história da educação, Lisboa.

Feix, E. y Goellner, S. V. (2008). O florescimento dos espaços públicos de lazer e de recreação em Porto Alegre e o protagonismo de Frederico Guilherme Gaelzer. Licere, 11(3), 1-18.

Frost, J. L. y Woods, I. C. (1998). Perspectives on play in playgrounds. En D. P. Fromberg y D. Bergen. Play from birth to twelve and beyond: contexts, perspectives, and meanings (pp. 232-240). Garland.

Freyre, G. (1979). Tempo de aprendiz. Artigos publicados em jornais na adolescência e na primeira mocidade do autor (1918-1926). Ibrasa/INL.

Freyre, G. (2006). Tempo morto e outros tempos: trechos de um diário de adolescência e primeira mocidade 1915-1930. Global.

Ginzburg, C. (2007). O fio e os rastros: verdadeiro, falso, fictício. Companhia das Letras.

Gomes, C. L. Significados de recreação e lazer no Brasil: reflexão a partir da análise de experiências institucionais (1926-1964). [Tesis de doctorado, Universidade federal de Minas Gerais]. Repositorio institucional UFMG http://hdl.handle.net/1843/HJPB-5NVJWV

Gulick, Luther H. (1909). Folk and national dances. En: Playground Association of America. Proceedings of the second annual Playground Congress and Year Book 1908 (pp. 429-439). Playground Association of America.

Hansan, J. Playground and Recreation Association of America (1925). The beginning of the recreation movement in the United States. Social Welfare History Project, 2013. http://socialwelfare.library.vcu.edu/ youth/recreation-movement-in-the-united-states/.

Ippolito, N. (1948). Educação sanitária. Boletim Interno da Divisão de Educação, Assistência e Recreio, 2(6), 120-121.

Kuhlmann Jr., M. (2019). Parque infantil: a singularidade e seus componentes. Educar em Revista, 35(77), 223-244.

Kuhlmann Jr., M. y Fernandes, F. S. (2014). Educação, cultura e infância no Parque Infantil paulistano (1947-1957). Diálogo Educacional, 14(43), 693-716.

Kuhlmann Jr, M. y Leonardi, P. (2017). História da educação no quadro das relações sociais. História da Educação, Santa Maria, 21(51), 207-227.

Luc, J.-N. La diffusion des modèles de préscolarisation em Europe dans la première moitié du XIX ${ }^{e}$ siècle. Histoire de l'éducation. (82), 1999, 189-206.

Moncorvo Filho, A. (1917). Os primeiros ensaios de heliotherapia no Brasil: trabalho do Dispensario Moncorvo. Typ. Besnard Frères. 
Moncorvo Filho, A. (1924) Em favor das creanças: a cura pelo sol. Typ. Besnard Frères.

Mooney-Melvin, P. (1983) Building muscles and civics: folk dancing, ethnic diversity and the Playground Association of America. American Studies, 24(1), 89-99.

Miranda, N. (1941). Origem e propagação dos parques infantis e parques de jogos. Departamento de Cultura.

Playground Association of America (1909). Proceedings of the second annual Playground Congress and Year Book 1908. Playground Association of America.

Scarlatto García, I. (2015). Corpo e tempo livre: as Plazas Vecinales de Cultura Física em Montevidéu (1911-1915). [Tesis de maestria, Universidade Federal de Santa Catarina]. Repositorio institucional UFSC. https://repositorio.ufsc.br/xmlui/handle/123456789/158909

Soares, C. L. (2016). Três notas sobre natureza, educação do corpo e ordem urbana (1900-1940). En: C. L., Soares (Org.). Uma educação pela natureza (pp. 9-45). Autores Associados.

Stewart, S. T. (1907). Kinder-Welten. The Playground, 1(4), 7-10.

Thompson, E. P. As peculiaridades dos ingleses e outros artigos. Editora da Unicamp.

Wilderspin, S. (1823). On the importance of educating the infant children of the poor. Goyder.

Wilderspin, S. (1852). The infant system for developing the intellectual and moral powers of all children from one to seven years of age. J. S. Hodson.

Wilderspin, S. (1828). Über die frühzeitige Erziehung der Kinder und die englischen Klein-Kinder-Schulen. Übersetzt von J. Wertheimer. Wien. https://books.google.com.br/books?id=8TVNAAAAcAAJ\&printsec=-

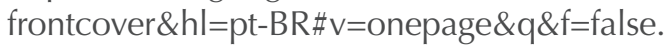

Williams, R. (1992). Cultura. Paz e Terra. 